\title{
Analysis of Risk Factors for Urinary Tract Infection in Community Inpatients with Extended-spectrum $\beta$-lactamase-producing Escherichia Coli
}

\author{
Xiaozhi Yang ${ }^{1,}$, , Jialin Guo ${ }^{1, b}$ \\ ${ }^{1}$ Luohe Medical College, Luohe, Henan, 462000 \\ ${ }^{\mathrm{a}}$ email, ${ }^{\mathrm{b}}$ email
}

Keywords: Urinary Tract Infection; Escherichia Coli; Extended-Spectrum B-lactamase

\begin{abstract}
To investigate the detection of extended-spectrum $\beta$-lactamases (ESBLs) -based Escherichia coli and their associated risk factors in urinary tract infection in patients with urinary tract infection. Methods The clinical data of 169 patients with escherichia coli infection and ESBLs were analyzed retrospectively. The risk factors for urinary tract infections in ESBLs-producing ESBLs-producing inpatients were analyzed by logistic regression analysis. Results Univariate analysis showed that there was significant difference $(\mathrm{P}<0.05)$ in benign prostatic hyperplasia, hospitalization days, indwelling catheterization, and third-generation cephalosporins in ESBLs group and non-ESBLs group. The logistic regression analysis showed that the third-generation cephalosporins $(\mathrm{OR}=4.361)$ was the risk factor for urinary tract infection in hospitalized patients with ESBLs-producing Escherichia coli. Conclusion Inpatients with indwelling catheterization and third-generation cephalosporins are more likely to develop urinary tract infections due to ESBLs-producing Escherichia coli. Patients with these risk factors should pay more attention to the indications of indwelling catheterization and reasonable The use of third-generation cephalosporins to reduce community-based hospitalized patients with ESBLs-producing Escherichia coli urinary tract infection.
\end{abstract}

\section{Introduction}

Urinary tract infection is pathogenic bacteria in the urinary tract and urine growth and reproduction, and violations of urinary tract mucosa and tissue caused by infectious diseases. ESBLs-producing Escherichia coli is one of the major pathogens causing urinary tract infections. ESBLs are a class of $\beta$-lactamases that can hydrolyze antibiotics such as penicillin, cephalosporins and monocyclic compounds. Amidase, which is characterized by multi-drug resistance to antibiotics, and through plasmid-mediated, in the same species or heterologous spread of bacteria, resulting in increased nosocomial infections,

This has been clinically concerned. In recent years, ESBLs-producing Escherichia coli-induced urinary tract infection in hospitalized patients increased year by year to understand the community hospitalized patients with ESBLs-producing Escherichia coli-induced urinary tract infection-related risk factors for the prevention and control of infection, rational use of antibiotics is important. In this study, 169 hospitalized patients with ESBLs positive and negative Escherichia coli infection were enrolled in this study. Multivariate logistic regression analysis was performed to explore the risk factors.

\section{Materials and methods}

Clinical data. Collected from January 2011 to October 2014 Shanghai Longhua Street Community Health Service Center and Dapuqiao Community Community Health Service Center for treatment of hospitalized patients with urinary tract infection in 338 cases of clean urine culture results are positive for Escherichia coli, While ESBLs detection. A total of 169 patients with ESBLs colorectal were selected by random number table

Escherichia coli caused by urinary tract infection in 100 cases (ESBLs group), non-ESBLs 
Escherichia coli induced urinary tract infection in 69 cases (non-ESBLs group).

Diagnostic criteria. Bacterial urinary tract infection diagnostic criteria: (1) clean the middle of the quantitative culture of bacteria or bacteria cocci. (2) patients with urinary tract irritation, fever, bacteremia and so on. (3) a pyuria (urinary sediment microscopic examination of white blood cells $>5$ / HP). With a diagnosis can have a clear manifestation of urinary tract infection and accompanied by significant pyuria, clean urine bacteria were also diagnosed as bacterial urinary tract infection.

Identification of bacteria. Clean the middle of the urine collected after the uniform sent to the Hailan Wei medical tests were detected in accordance with the bacterial culture and separation of the "National Clinical Laboratory Procedures" for the French bioMérieux Vitek2 microbial analyzer for identification of bacteria, ESBLs-producing strains were identified by the disk diffusion confirmation method recommended by the American Society for Clinical and Laboratory Standards (CLSI).

Analysis of indicators. The clinical data of 15 patients who may affect ESBLs-producing Escherichia coli infection in community-based inpatients were collected including sex, age, diabetes, benign prostatic hyperplasia, renal calculus, chronic renal failure, tumor, renal cyst, hospitalization days , Had admitted ICU or emergency, indwelling catheterization, cystoscopy, glucocorticoid use, the application of the third generation of the first Sporozoin history, urinary system surgery.

Statistical Methods. Clinical data were compared with SPSS 17. 0 statistical software, count data to test, and the parameters of multivariate logistic regression analysis, $\mathrm{P}<0.05$ for the difference was statistically significant.

\section{Results}

Univariate analysis. Prostatic hyperplasia, hospital stay $\geqslant 1$ month, indwelling catheterization, the application of third-generation cephalosporins in the proportion of ESBLs group than non-ESBLs group, the difference was statistically significant (Table 1).

Multivariate logistic regression analysis. ( 1 for men, 0 for women), age ( 1 for $\geqslant 65$ years, 0 for $<65$ years), diabetes, benign prostatic hyperplasia, kidney stones ( 1 for yes, 0 for no) ( $1=$ yes, 0 $=$ no), hospitalization time ( $1 \geqslant 1$ month, $0<1$ month), ICU or emergency room stay, catheterization, cystoscopy, Glucocorticoid use of the third-generation cephalosporin history, urinary system surgery ( 1 yes, 0 is no) and other factors as regression analysis of independent variables, the use of stepwise logistic regression analysis to establish the model, and finally stay

(X1), two independent variables of the third generation cephalosporin history (X2) were used to enter the model (Table 2). Regression equation significance test results: $=45.527, \mathrm{P}=0.00$.

\section{Discussion}

Escherichia coli is the main pathogen causing urinary tract infection in recent years with the growing bacterial resistance in hospitalized patients ESBLs-producing strains of urinary tract infections gradually increased, and easily lead to hospital outbreaks, community hospitals because of diagnosis and treatment Condition is limited, the treatment is faced with great difficulties, poor prognosis, high mortality. However, ESBLs-producing Escherichia coli in community-based hospitalized patients with urinary tract infection risk factors are not many reports, this paper by retrospective analysis of ESBLs-producing Escherichia coli-induced community hospitalization

Risk factors are for urinary tract infection in order to strengthen the monitoring and management of high-risk groups for more effective prevention and control of ESBLs-producing Escherichia coli to provide the basis for urinary tract infection. In this paper, logistic regression analysis showed that indwelling catheterization, the application of third-generation cephalosporins are caused by community-based patients with ESBLs-producing Escherichia coli urinary tract infection risk factors. Studies have shown that indwelling catheter for ESBLs-producing Escherichia coli urinary tract infection will also be affected. The results of this study show that patients with indwelling 
catheter more prone to ESBLs-producing Escherichia coli urinary tract infection, the risk is not indwelling catheter 4. 546 times. Indwelling catheter easily lead to a certain degree of mechanical damage to the urethra, destruction of the normal physiological barrier, increase the chances of infection. According to statistics, a catheterization after persistent bacteriuria occurred in $1 \%$ to $2 \%$; indwelling catheterization $4 \mathrm{~d}$ or more, the persistent bacteriuria rate was $90 \%$. Therefore, clinical indwelling catheterization indications should be strictly controlled indwelling catheterization of patients, it is recommended early exercise bladder voiding function, early removal, in order to reduce the chance of urinary tract infection.

Domestic and foreign studies have confirmed that the application of third-generation cephalosporins and ESBLs-producing Escherichia coli urinary tract infection are closely related. The results of this study show that the use of third-generation cephalosporins in community-based hospitalized patients with ESBLs-producing Escherichia coli urinary tract infection risk is 4.361 times the unused. Third-generation cephalosporins are broad-spectrum antibiotics that can induce the production of ESBLs by bacteria and can be spread between bacteria through plasmids. Therefore, the widespread use of third-generation cephalosporins and the use of antibiotics unreasonable phenomenon of ESBLs-producing Escherichia coli urinary tract infections have an important impact, suggesting the need to strengthen the supervision of the use of antibiotics, to avoid long-term application of third-generation cephalosporins Or similar frequent replacement, in order to reduce the production of ESBLs Escherichia coli production and dissemination.

\section{References}

[1] Ostrowska K, Strzelczyk A, Rózalski A, et al. Bacterial biofilmas a cause of urinary tract infection-pathogens, methods of prevention and eradication [J]. Postepy Hig Med Dosw (Online), 2013, 67: 1027 -1033.

[2] Hammami S, Saidani M, Ferjeni S, et al. Characterization of extended spectrum $\beta$-lactamase-producing Escherichia coli incommunity-acquired urinary tract infections in Tunisia [J]. Microb Drug Resist, 2013, 19 (3): 231-236.

[3] Qiao Ludong, Chen Shan, Meng Lihui. Analysis of risk factors of urinary tract infection in Escherichia coli producing ESBLs [J]. Chinese Journal of Nosocomiology, 2011,21 (2): 247 249.

[4] Chenoweth CE, Saint S. Urinary tract infections [J]. Infect DisClin North Am, 2011, 25 (1): 103-115.

[5] Chen Haozhu. Practical Internal Medicine [M]. 13 version. Beijing: People's Medical Publishing House, 2005: 2218.

[6] Tang Chunbo, Qi Yong. Cox proportional hazards model analysis of ESBLs Escherichia coli caused by urinary tract infection risk factors [J]. Chinese Journal of General Practitioners, 2014,12 (3): 397 - 399. 